This is a repository copy of An LMI-Based Design Method for Modular Observers in the Depósito de Investigación de la Universidad de Sevilla

Version: Author Accepted Version

Citation: An LMI-Based Design Method for Modular Observers. J. G. Martin, F. J. Muros, E. Masero, E. F. Camacho, J. M. Maestre. 2020 European Control Conference (ECC).

10.23919/ecc51009.2020.9143672

To cite this publication, please use the final published version (if applicable). Please check the document version above.

Copyright: Other than for strictly personal use, it is not permitted to download, forward or distribute the text or part of it, without the consent of the author(s) and/or copyright holder(s), unless the work is under an open content license such as Creative Commons.

Takedown policy: Please contact us (idus@us.es) and provide details if you believe this document breaches copyrights. We will remove access to the work immediately and investigate your claim 


\title{
An LMI-Based Design Method for Modular Observers*
}

\author{
J. G. Martin, F. J. Muros, E. Masero, E. F. Camacho and J. M. Maestre
}

\begin{abstract}
A linear matrix inequality approach for designing a family of observers suitable for systems with variable communication topologies is presented. In particular, the observer is composed of blocks associated to the status of the communication links, providing increasing performance as more links are enabled. The error boundaries for topology switchings are analyzed both for the presented observer and for a specific Kalman filter for each topology. Finally, a simulation example is used to illustrate the feasibility of the proposed method.
\end{abstract}

\section{INTRODUCTION}

In the last decades, distributed schemes have gained relevance in the field of control and estimation due to their wellknown advantages such as scalability, modularity, ease of implementation, and robustness, which make them appropriate for large-scale networked systems [1], and other complex approaches [2]. Distributed architectures are characterized for being composed of a set of subsystems, governed by agents that have access to local information. Consequently, communication is needed to gather information from the rest of the system to carry out estimation and control tasks in the most efficient way [3]. Reshaping the topology of the communication network according to the necessities of the system provides additional flexibility to optimize performance. In this way, only communication channels that really contribute to the improvement of the task are enabled (see, e.g., [4], [5] for cooperative approaches).

Through the observers, variables such as the system state, disturbances, noises, etc., can be estimated in the cases in which they cannot be measured directly [6]. Some classical estimators, e.g., the Luenberger filter [7], settled the basis for most observers designed nowadays. Also, the Kalman filter, which introduced white noise in the filtering formulation to offer optimal solutions [8], [9], has been widely studied and extended over the years [10]. Recently, both approaches have been adapted to noncentralized systems requirements. Some distributed Luenberger approaches for linear systems are explored in: [11], with a given fixed communication architecture between subsystems; [12], where time-varying communication topologies are considered; and [13], where consensus between subsystems is used to estimate the nonlocally detectable portion of the state. More details about

*This work has been funded by the European Research Council (ERC) under the Advanced Grant OCONTSOLAR (grant agreement number 789051), the Spanish Ministry of Economy (project ref. DPI201786918-R), and the Spanish Ministry of Science, Innovation and Universities (Ph.D. grant FPU18/04476).

J. G. Martin, F. J. Muros, E. Masero, E. F. Camacho and J. M. Maestre are with the Department of Systems and Automation Engineering, University of Seville, Spain \{jgarmar, franmuros, evamasero, efcamacho, pepemaestre\} dus.es distributed observers can be consulted in [14]. Regarding to Kalman filters, in [15], a decentralized Kalman filter is used to address the localization of a multiple-robot problem; [16] discusses three distributed Kalman filter algorithms, one of them focused on a cooperative and recursive estimation that combines the consensus strategy and Kalman filtering; [17] focuses on the scheduling of data in a networked system; and [18] develops a method based on linear matrix inequalities (LMIs) to compute the Kalman filter, being this method implemented in [19] for multi-robot localization purposes. Note that LMIs have become a major way of formulating a vast variety of control and estimation problems [20]. In particular, the development of LMI control toolboxes for software environments as Matlab ${ }^{\circledR}$ [21] has increased the use of LMIs in several applications, e.g., [22]-[24].

Generally, the reconfiguration of the communication topology in distributed observer approaches [25], [26] leads to an increase in the computational cost due to the combinatorial explosion of the possibilities. Furthermore, the implementation of these methods on real systems brings several problems. To begin with, each observer must store as many configurations as topologies are taken into account. In addition, an upper layer may be necessary in the estimation architecture for the sake of coordination. With the idea of tackling these issues, this paper proposes a novel observer, in the line of [18], which, by contrast, is suitable for all the topologies considered. More specifically, the observer presented in this paper has a modular structure, i.e., it is composed of fixed blocks that are assigned to the status of the communication links. The value of the elements inside these blocks has a constant value for all the topologies where the corresponding link is activated and becomes zero otherwise. Consequently, a single observer is designed for all topologies, thus greatly simplifying the implementation of the observer and removing the need for a coordination layer. Nevertheless, extra restrictions need to be introduced in the problem to guarantee that it works with the desired set of topologies with a modular structure. For this reason, the proposed method becomes more conservative than a classical Kalman filter. Hence, an additional goal of this work is to assess the modular observer with other methods such as a topology specific Kalman filter.

The rest of the paper is organized as follows. Section II presents the problem formulation. Section III introduces the concept of modular observer, providing the LMI-based design method and some properties of interest. Section IV illustrates the proposed method through an academic example. Finally, conclusions are detailed in Section V. 


\section{PROBLEM FORMULATION}

Consider an uncertain distributed linear system composed by a set of agents $\mathcal{N}=\{1,2, \ldots, n\}$, where the discrete-time model of a single agent $i \in \mathcal{N}$ is described by

$$
\begin{aligned}
x_{i}(k+1) & =A_{i i} x_{i}(k)+\sum_{j \in \mathcal{N}_{i}} A_{i j} x_{j}(k)+Q_{i} w_{i}(k), \\
y_{i}(k) & =C_{i i} x_{i}(k)+\sum_{j \in \mathcal{N}_{i}} C_{i j} x_{j}(k)+R_{i} v_{i}(k),
\end{aligned}
$$

where $x_{i} \in \mathbb{R}^{n_{i}}$ and $x_{j} \in \mathbb{R}^{n_{j}}$ are respectively the state vectors of subsystem $i$ and its neighboring subsystem $j, \mathcal{N}_{i}$ denotes the set of neighbors of agent $i$, i.e., $\mathcal{N}_{i}:=\{j \in$ $\left.\mathcal{N}, i \neq j: A_{i j} \neq 0 \vee C_{i j} \neq 0\right\} ; y_{i} \in \mathbb{R}^{q_{i}}$ refers to the corresponding output vector, and $w_{i} \in \mathbb{R}^{n_{i}}$ and $v_{i} \in \mathbb{R}^{q_{i}}$ are normal noises with zero mean and unit standard deviation, i.e., $\mathscr{N}(0,1)$. Likewise, $A_{i i} \in \mathbb{R}^{n_{i} \times n_{i}}$ and $C_{i i} \in \mathbb{R}^{q_{i} \times n_{i}}$ are respectively the state and output matrices of subsystem $i$, and matrices $A_{i j} \in \mathbb{R}^{n_{i} \times n_{j}}$ and $C_{i j} \in \mathbb{R}^{q_{i} \times n_{j}}$ are related to the interrelation between subsystems. Finally, $Q_{i} \in \mathbb{R}^{n_{i} \times n_{i}}$ and $R_{i} \in \mathbb{R}^{q_{i} \times q_{i}}$ are diagonal matrices that describe the magnitude of the noise.

The dynamics of the global system, which is assumed to be observable, are given by

$$
\begin{aligned}
x_{\mathcal{N}}(k+1) & =A_{\mathcal{N}} x_{\mathcal{N}}(k)+Q_{\mathcal{N}} w_{\mathcal{N}}(k), \\
y_{\mathcal{N}}(k) & =C_{\mathcal{N}} x_{\mathcal{N}}(k)+R_{\mathcal{N}} v_{\mathcal{N}}(k),
\end{aligned}
$$

where the aggregate vectors and matrices are described by $x_{\mathcal{N}}=\left[x_{i}\right]_{i \in \mathcal{N}}, y_{\mathcal{N}}=\left[y_{i}\right]_{i \in \mathcal{N}}, A_{\mathcal{N}}=\left[A_{i j}\right]_{i, j \in \mathcal{N}}, C_{\mathcal{N}}=$ $\left[C_{i j}\right]_{i, j \in \mathcal{N}}, Q_{\mathcal{N}}=\operatorname{diag}\left(Q_{i}\right)_{i \in \mathcal{N}}, R_{\mathcal{N}}=\operatorname{diag}\left(R_{i}\right)_{i \in \mathcal{N}}, w_{\mathcal{N}}=$ $\left[w_{i}\right]_{i \in \mathcal{N}}$ and $v_{\mathcal{N}}=\left[v_{i}\right]_{i \in \mathcal{N}}$.

The overall estimated state $\hat{x}_{\mathcal{N}}(k)$ can be calculated from (2) as

$$
\hat{x}_{\mathcal{N}}(k)=A_{\mathcal{N}} \hat{x}_{\mathcal{N}}(k-1)+L\left(y_{\mathcal{N}}(k)-C_{\mathcal{N}} A_{\mathcal{N}} \hat{x}_{\mathcal{N}}(k-1)\right),
$$

with $L$ being the observer matrix for the global system.

\section{A. Error bounds}

The error is defined as the difference between the overall state and its estimation, i.e.,

$$
e_{\mathcal{N}}(k)=x_{\mathcal{N}}(k)-\hat{x}_{\mathcal{N}}(k) .
$$

From (2), (3) and (4), we can obtain

$$
e_{\mathcal{N}}(k+1)=A_{p} e_{\mathcal{N}}(k)+Q_{p} w_{\mathcal{N}}(k)+R_{p} v_{\mathcal{N}}(k+1),
$$

where $A_{p}=\left(A_{\mathcal{N}}-L C_{\mathcal{N}} A_{\mathcal{N}}\right), Q_{p}=\left(Q_{\mathcal{N}}-L C_{\mathcal{N}} Q_{\mathcal{N}}\right)$ and $R_{p}=L R_{\mathcal{N}}$.

Note that (5) can be considered as a dynamical system with two inputs, i.e., $w_{\mathcal{N}}$ and $v_{\mathcal{N}}$. This system will be stable as long as $A_{p}$ is stable, or in other words, as long as the eigenvalues of $A_{p}$ are inside the unit circle.

Defining $B_{p}=\left[\begin{array}{ll}Q_{p} & R_{p}\end{array}\right]$, and $u_{\mathcal{N}}(k)=$ $\left[\begin{array}{ll}w_{\mathcal{N}}(k)^{\mathrm{T}} & v_{\mathcal{N}}(k+1)^{\mathrm{T}}\end{array}\right]^{\mathrm{T}}$, the error system in (5) can be rewritten as

$$
e_{\mathcal{N}}(k+1)=A_{p} e_{\mathcal{N}}(k)+B_{p} u_{\mathcal{N}}(k) .
$$

At this point, applying (6) recursively, it is possible to define the aggregate input vector along a given window of arbitrary length $N$ as $U=\left[\begin{array}{llll}u_{\mathcal{N}}(k-N) & u_{\mathcal{N}}(k-N+1) & \cdots & u_{\mathcal{N}}(k-1)\end{array}\right]^{\mathrm{T}}$. Hence, the current error can be rewritten as

$$
e_{\mathcal{N}}(k)=G_{e} e_{\mathcal{N}}(k-N)+G_{u} U,
$$

where $G_{e}=A_{p}^{N}$ and the input matrix is $G_{u}=$ $\left[\begin{array}{lllll}A_{p}^{N-1} B_{p} & A_{p}^{N-2} B_{p} & \cdots & A_{p} B_{p} & B_{p}\end{array}\right]$.

Since $A_{p}$ is considered stable, there must be a value of $N$ large enough that makes the first term in (7) vanish, i.e., that corresponding to the error in the system at instant $k-N$, which is assumed to be finite. Therefore, we can rewrite the system error at time instant $k$ as

$$
e_{\mathcal{N}}(k)=\sum_{i=1}^{N} A_{p}^{N-i} B_{p} u_{\mathcal{N}}(k-N-1+i) .
$$

Provided that $u_{\mathcal{N}}(k)$ is a vector composed of normal variables $\mathscr{N}(0,1)$ for every $k$, we can obtain $e_{\mathcal{N}}(k)$ as a normal variable $\mathscr{N}\left(0, \sigma_{e}\right)$. Hence, the error can be bounded to a known certainty by using the well-known expression [27]

$$
P\left(a \leq e_{\mathcal{N}} \leq b\right)=\frac{1}{\sqrt{2 \pi \sigma_{e}^{2}}} \int_{\mathrm{a}}^{b} \exp \left(\frac{-e_{\mathcal{N}}^{2}}{2 \sigma_{e}^{2}}\right) d e_{\mathcal{N}},
$$

which will be considered in the simulation results.

\section{B. Communication constraints}

We can describe the communication network as a graph $(\mathcal{N}, \mathcal{L})$ where $\mathcal{N}$ and $\mathcal{L}$ refer respectively to the set of nodes or agents of the system, and the set of possible communication links connecting the nodes. Note that $\mathcal{L} \subseteq$ $\mathcal{L}^{\mathcal{N}}=\{\{i, j\} \mid\{i, j\} \subseteq \mathcal{N}, i \neq j\}$. It is assumed that there is only direct communication, i.e., node $j$ will get information from node $i$ iff $\ell_{i j}$ is enabled. Let us define $\Lambda$ as a given topology of enabled communication links. Then, it is possible to introduce $\mathcal{T}=\left\{\Lambda_{\mathrm{DC}}, \Lambda_{1}, \Lambda_{2}, \ldots, \Lambda_{\mathcal{L}}\right\}$ as the set of all the possible topologies of our system, where $\Lambda_{\mathrm{DC}}$ corresponds to the totally decentralized topology (no communication links enabled) and $\Lambda_{\mathcal{L}}$ refers to the totally centralized system (every node has information from all the other nodes).

To illustrate this fact, consider for instance the system represented in Fig. 1, which is composed of four agents and consider all the possible connections between them. For this

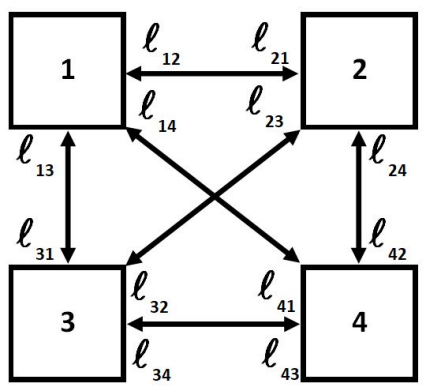

Fig. 1. Different communication links for a 4-agent system 
scenario, it is possible to define the following observer:

$$
L=\left[\begin{array}{llll}
L_{11} & L_{12} & L_{13} & L_{14} \\
L_{21} & L_{22} & L_{23} & L_{24} \\
L_{31} & L_{32} & L_{33} & L_{34} \\
L_{41} & L_{42} & L_{43} & L_{44}
\end{array}\right]
$$

where $L=\left[L_{i j}\right]_{i, j \in \mathcal{N}}$ if $\ell_{i j}$ is enabled and $L_{i j}=0$ if $\ell_{i j}$ is disabled.

According to (10), observer $L^{\Lambda_{\mathcal{L}}}$ for the centralized topology would have the complete structure presented in (10), whereas $L^{\Lambda_{\mathrm{DC}}}$ would have the following form:

$$
L^{\Lambda_{\mathrm{DC}}}=\left[\begin{array}{cccc}
L_{11} & 0 & 0 & 0 \\
0 & L_{22} & 0 & 0 \\
0 & 0 & L_{33} & 0 \\
0 & 0 & 0 & L_{44}
\end{array}\right]
$$

Likewise, assuming an intermediate topology, e.g., the case where node 2 is completely isolated and the rest of nodes are connected, the corresponding observer, say $L^{\Lambda_{\mathrm{ex}}}$, would have the following structure:

$$
L^{\Lambda_{\mathrm{ex}}}=\left[\begin{array}{cccc}
L_{11} & 0 & L_{13} & L_{14} \\
0 & L_{22} & 0 & 0 \\
L_{31} & 0 & L_{33} & L_{34} \\
L_{41} & 0 & L_{43} & L_{44}
\end{array}\right] .
$$

Remark 1: For each observer $L^{\Lambda}, \forall \Lambda \in \mathcal{T}$, all nonzero elements have the same value, so that the observer defined in (10) becomes actually a family of observers that can be adapted to each topology simply by making zero the corresponding blocks.

\section{MODULAR OBSERVER DESIGN}

In this section, a method that refines that of [18] is provided to design the modular observer. In particular, the observer in [18] was suitable for a static network configuration. By contrast, extra restrictions have been added here, by means of the following theorem, to guarantee modularity, i.e., to allow the observer to be implementable for the full set of topologies:

Theorem 1 Let the system be described by $A_{\mathcal{N}}$ and $C_{\mathcal{N}}$, and the disturbances be characterized by normal noise with covariance matrices $Q_{\mathcal{N}}$ and $R_{\mathcal{N}}$. If there exist matrices $W_{\mathcal{N}}=W_{\mathcal{N}}^{\mathrm{T}}=\operatorname{diag}\left(W_{i}\right)_{i \in \mathcal{N}}$, with $W_{i} \in \mathbb{R}^{n_{i} \times n_{i}}$ and $S^{\Lambda}=$ $\left[S_{i j}^{\Lambda}\right]_{i, j \in \mathcal{N}}$, with $S_{i j}^{\Lambda} \in \mathbb{R}^{n_{i} \times q_{j}}$ if $\ell_{i j}$ is enabled and $S_{i j}^{\Lambda}=0$ if $\ell_{i j}$ is not enabled, such that the LMI

$$
\left[\begin{array}{cccc}
-W_{\mathcal{N}} & W_{\mathcal{N}} A_{\mathcal{N}}-S^{\Lambda} C_{\mathcal{N}} A_{\mathcal{N}} & W_{\mathcal{N}}-S^{\Lambda} C_{\mathcal{N}} & S^{\Lambda} \\
* & -W_{\mathcal{N}} & 0 & 0 \\
* & * & -Q_{\mathcal{N}}^{-1} & 0 \\
* & * & * & -R_{\mathcal{N}}^{-1}
\end{array}\right]<0,
$$

has a feasible solution for all $\Lambda \in \mathcal{T}$, then there exists a family of modular observers provided by $L^{\Lambda}=W_{\mathcal{N}}^{-1} S^{\Lambda}$ that have the same bound in the steady-state error covariance matrix, given by $P_{\mathcal{N}}=W_{\mathcal{N}}^{-1}$.

The proof of Theorem 1 is straightforward following [18]. In any case, note that the set of topologies $\mathcal{T}$ does not need to be exhaustive, i.e., it can be composed only by a reduced set of topologies of interest, say $\mathcal{F}$. Moreover, the following lemma introduces a further way in which the design of the observer can be simplified to reduce the issues derived from the combinatorial explosion in the number of topologies.

Lemma 1 Consider a family of topologies $\mathcal{F}_{\mathrm{a}}$ composed of a topology $\Lambda_{\mathrm{a}}$ with a set of enabled communication links, and all its parent topologies, which are those with at least the same set of enabled communication links than $\Lambda_{\mathrm{a}}$. Iff there exists a feasible solution following (13) for $\Lambda_{\mathrm{a}}$, there is also a feasible solution for any $\Lambda \in \mathcal{F}_{\mathrm{a}}$ and hence, a modular observer $L_{\mathcal{F}_{\mathbf{a}}}$ for the complete family $\mathcal{F}_{\mathrm{a}}$ can be found.

Notice that the condition established in Lemma 1 is necessary given that (13) needs to be satisfied for $\Lambda_{\mathrm{a}}$. Likewise, it is sufficient since a feasible solution of (13) for $\Lambda_{\mathrm{a}}$ is also a feasible solution for any of its parents topologies. In particular, note that the solution for $\Lambda_{\mathrm{a}}$ corresponds to the particular solution for its parents topologies where the decision variables that correspond to the disabled links blocks are set to zero. Consequently, if there is no solution for $\Lambda_{\mathrm{a}}$, there cannot be a solution for the modular problem for family $\mathcal{F}_{\mathrm{a}}$.

Remark 2: The result of Lemma 1 can be particularized for the decentralized case, i.e., $\Lambda_{\mathrm{a}}=\Lambda_{\mathrm{DC}}$. If there is a solution for $\Lambda_{\mathrm{DC}}$ in (13), there is a feasible solution for every topology and hence, a modular observer for all $\Lambda \in \mathcal{T}$.

While it may seem restrictive requiring the topology with the fewest links to have a solution, note that it is unavoidable because the system also needs to work under that topology. Likewise, note that the performance of the observer improves as more links become available, as it will be shown with the numerical example in the next section.

\section{A. Design Method}

The observer can be designed by solving

$$
\max _{W_{\mathcal{N}}, S^{\Lambda}} \operatorname{tr}\left(W_{\mathcal{N}}\right)
$$

subject to LMI (13) for all $\Lambda \in \mathcal{T}$. Maximizing the trace of matrix $W_{\mathcal{N}}$, leads to minimizing the trace of matrix $P_{\mathcal{N}}$, which in turn reduces the steady-state error. By imposing constraints for all topologies the solution space is restricted, so that the modular observer $L_{\mathcal{F}_{\mathrm{a}}}$ does not have the performance of a Kalman filter designed specifically for a topology $\Lambda \in \mathcal{F}_{\mathrm{a}}$. Nevertheless, the proposed modular observer gains ease of implementation and modularity with respect to the topology specific Kalman filter.

Remark 3: In a previous work [4], an optimization problem was solved for each topology, i.e., the LMI conditions related to each topology were computed independently. By contrast, here only one optimization problem is solved for all the topologies $\Lambda \in \mathcal{F}_{\mathrm{a}}$, by solving simultaneously the full set of LMIs described in (13), obtaining the family of modular observers $L_{\mathcal{F}_{\mathrm{a}}}$. In any case, combinatorial explosion issues are still present because the computation complexity of 
LMIs does not scale linearly with the number of constraints, as shown in [28]. Hence, this method is suitable for a modest number of agents if all topologies are to be considered. Another alternative to mitigate the complexity of the problem is by using a branch-and-bound-like approach [29] based on Lemma 1. In particular, a bound on the performance is given by that of the centralized Kalman filter. Also, a lower bound on the performance of any topology is provided by any of its sons, i.e., topologies with fewer activated links. Hence, it is possible to select which branches of topologies are more promising regarding a possible gain of performance. This is a matter of current research.

\section{AN ILLUSTRATIVE EXAMPLE}

In order to illustrate and assess the modular observer presented in this work, we take the following academic scheme, which consists of four subsystems whose discretetime dynamics are given by

$$
\begin{gathered}
A_{11}=\left[\begin{array}{ll}
0 & 0.5 \\
1 & 0.4
\end{array}\right], \quad A_{22}=\left[\begin{array}{ll}
0 & 0.3 \\
1 & 0.2
\end{array}\right], \\
A_{33}=\left[\begin{array}{cc}
0 & 0.4 \\
1 & 0.45
\end{array}\right], \quad A_{44}=\left[\begin{array}{ll}
0 & 0.8 \\
1 & 0.1
\end{array}\right], \\
A_{i j}=\left[\begin{array}{ll}
0 & 0 \\
0 & 0
\end{array}\right], i \neq j, \forall i, j \in \mathcal{N}, \\
C_{i j}=\left[\begin{array}{ll}
0 & 1
\end{array}\right], i=j, \forall i, j \in \mathcal{N}, \\
C_{i j}=\left[\begin{array}{ll}
0 & 0.15
\end{array}\right], i \neq j, \forall i, j \in \mathcal{N}, \\
Q_{i}=\left[\begin{array}{cc}
10 & 0 \\
0 & 10
\end{array}\right], \forall i \in \mathcal{N}, \quad R_{i}=[10], \forall i \in \mathcal{N},
\end{gathered}
$$

i.e., there is no coupling in the state evolution but agents can help others to improve their estimates.

For this example, four different topologies have been considered: the centralized and decentralized topologies, numbered as 1 and 4, respectively, and also two intermediate topologies - numbered as 2 and 3 -, as shown in Fig. 2. Notice that in each of these topologies the links that have been considered are bidirectional, i.e., if $l_{i j}$ is enabled, $l_{j i}$ is enabled as well. This family of topologies is denoted as $\mathcal{F}_{\text {a }}$ and, as stated in Lemma 1, there is a solution for the modular observer iff there is a solution for the decentralized topology, since it is the most restrictive topology of the family.
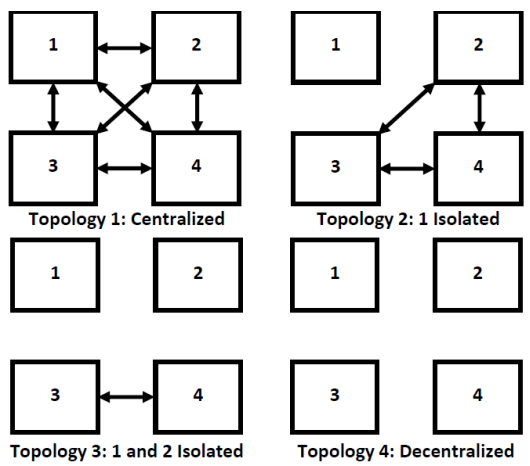

Fig. 2. Different topologies considered in the academic example
Considering the design method presented in Section III-A, the following overall matrix $P_{\mathcal{N}}$ has been obtained:

$$
P_{\mathcal{N}}=\left[\begin{array}{cccccccc}
12.3019 & 0.8017 & 0 & 0 & 0 & 0 & 0 & 0 \\
0.8017 & 8.9153 & 0 & 0 & 0 & 0 & 0 & 0 \\
0 & 0 & 10.9167 & 0.2276 & 0 & 0 & 0 & 0 \\
0 & 0 & 0.2276 & 8.4889 & 0 & 0 & 0 & 0 \\
0 & 0 & 0 & 0 & 11.5043 & 0.6927 & 0 & 0 \\
0 & 0 & 0 & 0 & 0.6927 & 8.8597 & 0 & 0 \\
0 & 0 & 0 & 0 & 0 & 0 & 16.1193 & 0.3588 \\
0 & 0 & 0 & 0 & 0 & 0 & 0.3588 & 9.1431
\end{array}\right]
$$

and the observer for the centralized topology is calculated by means of $L^{\Lambda}=W_{\mathcal{N}}^{-1} S^{\Lambda}$ as stated in Theorem 1, obtaining

$$
L_{\mathcal{F}_{\mathrm{a}}}^{\Lambda_{\mathcal{L}}}=\left[\begin{array}{cccc}
0.0666 & -0.0009 & -0.0005 & -0.0005 \\
0.7435 & -0.0100 & -0.0059 & -0.0061 \\
-0.0003 & 0.0191 & -0.0001 & -0.0002 \\
-0.0097 & 0.7073 & -0.0055 & -0.0057 \\
-0.0007 & -0.0007 & 0.0579 & -0.0004 \\
-0.0088 & -0.0085 & 0.7404 & -0.0053 \\
-0.0004 & -0.0003 & -0.0002 & 0.0297 \\
-0.0091 & -0.0087 & -0.0053 & 0.7654
\end{array}\right] .
$$

Let us consider for instance the observer for topology $\Lambda_{\mathrm{ex}}=3$ in Fig. 2, which is now simply formed by adding zeros in the blocks corresponding to nodes 1 and 2 in (17), leading to the observer

$$
L_{\mathcal{F}_{\mathrm{a}}}^{\Lambda_{\text {ex }}}=\left[\begin{array}{cccc}
0.0666 & 0 & 0 & 0 \\
0.7435 & 0 & 0 & 0 \\
0 & 0.0191 & 0 & 0 \\
0 & 0.7073 & 0 & 0 \\
0 & 0 & 0.0579 & -0.0004 \\
0 & 0 & 0.7404 & -0.0053 \\
0 & 0 & -0.0002 & 0.0297 \\
0 & 0 & -0.0053 & 0.7654
\end{array}\right] .
$$

That is, only one problem needs to be solved since the observers for the rest of topologies are directly derived from the centralized approach. Note that an LMI for each topology would have to be solved to obtain a topology specific Kalman filter, which in the worst case corresponds to $2^{|\mathcal{N}|}$ problems. Furthermore, due to the modular nature of the proposed observer, each agent only needs to know its column in $L^{\Lambda_{\mathcal{L}}}$. For example, agent 1 only needs to know the first column of the modular observer in (17). By contrast, in case we had topology dependant observers, the agent would need to know a different column per topology plus information relative to what topology is active. Hence, the modular approach allows simplifying the implementation of observers for systems with time-varying communication networks.

To test this modular observer, we present an experiment where we have considered 400 iterations, starting with the centralized topology, i.e., topology 1 , changing to the intermediate topology 2 at time step $k=100$, then to topology 3 at $k=200$, and finally to topology 4 (decentralized) at $k=$ 300, as shown in Fig. 3A, where connected and disconnected agents are represented in green and blue, respectively. In particular, we compare the results of the modular observer (MOD), with an observer consisting on different Kalman filters designed for each specific topology, here referred to as non-modular observer (NMOD), and also with the 
Kalman filter designed for the decentralized topology, which is denoted as decentralized observer (DEC).

As shown in red color in Fig. 3B, for the topologies where there is a high number of enabled communication links, square error $e_{\mathcal{N}}(k)^{\mathrm{T}} e_{\mathcal{N}}(k)$ in the MOD scheme is higher than that of the NMOD approach. Nevertheless, as long as the topology is closer to the decentralized one, this difference decreases. In contrast, when comparing the MOD and DEC approaches, the opposite occurs, which is depicted in blue. In fact, for topologies where there are more enabled links, the square error in the MOD scheme is lower than in the DEC scheme since the MOD observer uses extra information. In other words, the performance of the observer increases as new links become available. In order to quantify these differences, the integral square error (ISE) of the different observers have been calculated, resulting that the ISE $_{M O D}$ is $1.38 \%$ lower than the $\mathrm{ISE}_{\mathrm{DEC}}$ and it is only $4.4 \%$ higher than the ISE $E_{N M O D}$.

Note also that by using (8), and taking into account that both $A_{p}$ and $B_{p}$ depend on $L^{\Lambda}$ as can be seen in (5) and (6), it can be concluded that the error variance of the system, i.e., $\sigma_{e}$, depends on the observer being used, and hence, on the topology. For example, by establishing a $92 \%$ interval of confidence and using (9), we can calculate the boundaries of the error resulting in $1.75 \sigma_{e}$, which are satisfactory in our experiments, as drawn in Fig. 4 for the particular case of state 1 (the first state of agent 1 ).

Also, a full comparison of the error variance is detailed in Table I, where it can be seen that $\sigma_{e}$ decreases as more links are enabled in both MOD and NMOD schemes. We can also

A)

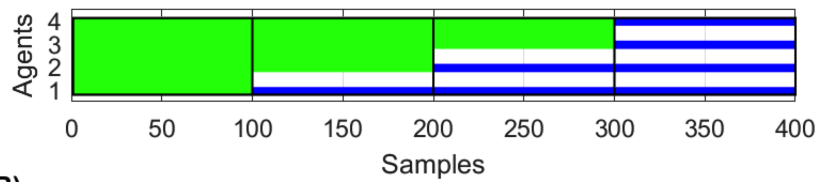

B)

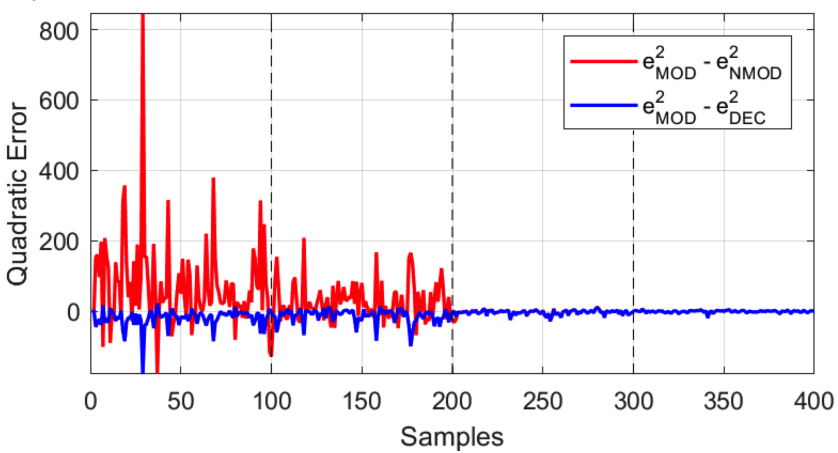

Fig. 3. Comparison between the square errors for the topologies in Fig. 2

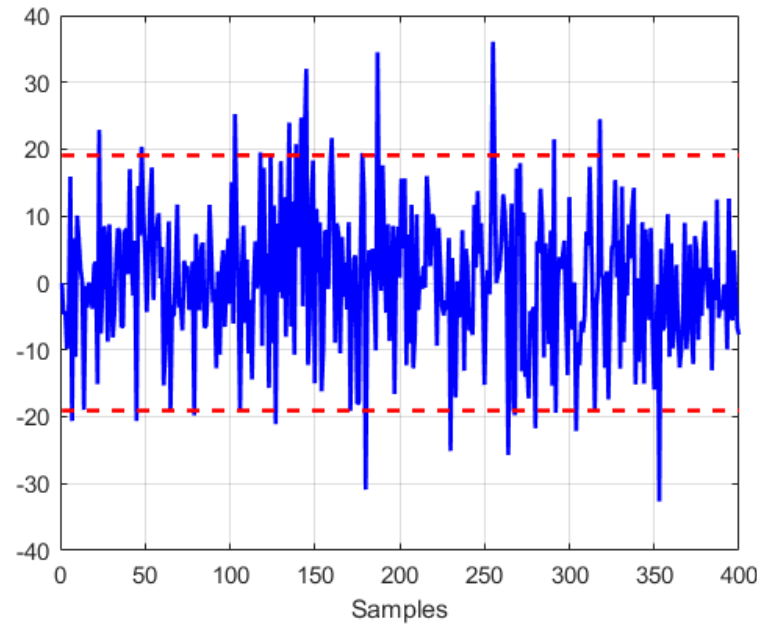

Fig. 4. Error in state 1 provided by the MOD approach

see that $\sigma_{e}$ decreases less in the MOD approach than in the NMOD scheme. Likewise, $\sigma_{e}$ converges for both observers for the decentralized topology.

\section{CONCLUSIONS}

In this paper, a novel observer with a modular structure, which consists of a family of observers suitable for a distributed system with variable communication topologies and based on linear matrix inequalities (LMIs), has been presented. To obtain this family, a single LMI problem needs to be solved instead of requiring the computation of one LMI per topology. Moreover, the modular structure simplifies considerably the implementation of local observers, which only need to know which agents they are receiving information from and, consequently, there is no need of an upper layer that compiles information regarding the current topology. Also, a theorem that guarantees modularity and a lemma showing a necessary and sufficient condition to design this type of observers have been proposed.

The feasibility of the proposed modular observer has been shown via an academical example and its results have been compared with those of a Kalman filter specialized for each topology, and also with the case of a decentralized Kalman filter. As it can be seen, the modular scheme outperforms the decentralized one and it is not far away from the specific Kalman filter for each topology, being the loss of accuracy strongly compensated with the ease of implementation of the proposed approach.

Future work should include the implementation of the proposed observer in a realistic large-scale network, including the use of branch-and-bound methods to mitigate the combinatorial explosion, in the line of what is commented in Remark 3. 
TABLE I

ERROR VARIANCE $\sigma_{e}$ OF EVERY STATE OF THE SYSTEM IN THE TOPOLOGIES INVOLVED IN THE EXPERIMENT

\begin{tabular}{l|cccccccc}
\hline MOD & $\sigma_{e_{1}}$ & $\sigma_{e_{2}}$ & $\sigma_{e_{3}}$ & $\sigma_{e_{4}}$ & $\sigma_{e_{5}}$ & $\sigma_{e_{6}}$ & $\sigma_{e_{7}}$ & $\sigma_{e_{8}}$ \\
\hline Topology 1 $\left(\Lambda_{\mathrm{DC}}\right)$ & 10.8979 & 8.8826 & 10.3306 & 8.6849 & 10.5754 & 8.8578 & 12.3022 & 8.9824 \\
Topology 2 (1 isolated) & 10.9079 & 8.9381 & 10.3323 & 8.7085 & 10.5781 & 8.8804 & 12.3132 & 9.0063 \\
Topology 3 ( 1 and 2 isolated) & 10.9079 & 8.9381 & 10.3344 & 8.7374 & 10.5804 & 8.9002 & 12.3229 & 9.0274 \\
Topology 4 $\left.\Lambda_{\mathcal{L}}\right)$ & 10.9079 & 8.9382 & 10.3344 & 8.7376 & 10.5822 & 8.9153 & 12.3296 & 9.0419 \\
\hline NMOD & $\sigma_{e_{1}}$ & $\sigma_{e_{2}}$ & $\sigma_{e_{3}}$ & $\sigma_{e_{4}}$ & $\sigma_{e_{5}}$ & $\sigma_{e_{6}}$ & $\sigma_{e_{7}}$ & $\sigma_{e_{8}}$ \\
\hline Topology 1 $\left(\Lambda_{\mathrm{DC}}\right)$ & 10.8590 & 8.6921 & 10.3177 & 8.5272 & 10.5502 & 8.6696 & 12.1962 & 8.7730 \\
Topology 2 $(1$ isolated) & 10.9969 & 9.3568 & 10.3234 & 8.5976 & 10.5607 & 8.7488 & 12.2398 & 8.8582 \\
Topology 3 $(1$ and 2 isolated) & 10.9104 & 8.9520 & 10.3353 & 8.7492 & 10.5826 & 8.9199 & 12.3308 & 9.0445 \\
Topology 4 $\left(\Lambda_{\mathcal{L}}\right)$ & 10.9098 & 8.9489 & 10.3351 & 8.7471 & 10.5828 & 8.9212 & 12.3314 & 9.0457 \\
\hline DEC & $\sigma_{e_{1}}$ & $\sigma_{e_{2}}$ & $\sigma_{e_{3}}$ & $\sigma_{e_{4}}$ & $\sigma_{e_{5}}$ & $\sigma_{e_{6}}$ & $\sigma_{e_{7}}$ & $\sigma_{e_{8}}$ \\
\hline Any topology & 10.9098 & 8.9489 & 10.3351 & 8.7471 & 10.5828 & 8.9212 & 12.3314 & 9.0457
\end{tabular}

\section{REFERENCES}

[1] A. N. Venkat, J. B. Rawlings, and S. J. Wright, "Distributed model predictive control of large-scale systems," in Assessment and Future Directions of Nonlinear Model Predictive Control. Springer, 2007, pp. 591-605.

[2] J. M. Maestre and R. R. Negenborn, Eds., Distributed model predictive control made easy. Springer, 2014, vol. 69.

[3] R. Scattolini, "Architectures for distributed and hierarchical model predictive control-a review," Journal of process control, vol. 19, no. 5, pp. 723-731, 2009.

[4] J. M. Maestre, D. Muñoz de la Peña, A. Jiménez Losada, E. Algaba, and E. F. Camacho, "A coalitional control scheme with applications to cooperative game theory," Optimal Control Applications and Methods, vol. 35, no. 5, pp. 592-608, 2014.

[5] F. Fele, J. M. Maestre, and E. F. Camacho, "Coalitional control: Cooperative game theory and control," IEEE Control Systems Magazine, vol. 37, no. 1, pp. 53-69, 2017.

[6] A. Radke and Z. Gao, "A survey of state and disturbance observers for practitioners," in Proceedings of the 24th IEEE American Control Conference, 2006, pp. 5183-5188.

[7] D. G. Luenberger, "Observing the state of a linear system," IEEE transactions on military electronics, vol. 8, no. 2, pp. 74-80, 1964.

[8] R. E. Kalman, "A new approach to linear filtering and prediction problems," Journal of basic Engineering, vol. 82, no. 1, pp. 35-45, 1960.

[9] R. E. Kalman and R. S. Bucy, "New results in linear filtering and prediction theory," Journal of basic engineering, vol. 83, no. 1, pp. 95-108, 1961.

[10] E. A. Wan and R. Van Der Merwe, "The unscented Kalman filter for nonlinear estimation," in Proceedings of the IEEE 2000 Adaptive Systems for Signal Processing, Communications, and Control Symposium, 2000, pp. 153-158.

[11] T. Kim, H. Shim, and D. D. Cho, "Distributed Luenberger observer design," in Proceedings of the IEEE 55th Conference on Decision and Control, 2016, pp. 6928-6933.

[12] W. Ni, X. Wang, J. Yang, and C. Xiong, "Distributed Luenberger observers for linear systems," in Proceedings of the 10th IEEE World Congress on Intelligent Control and Automation, 2012, pp. 4267-4271.

[13] A. Mitra and S. Sundaram, "Distributed observers for LTI systems," IEEE Transactions on Automatic Control, vol. 63, no. 11, pp. 36893704, 2018.

[14] F. F. Rego, A. M. Pascoal, A. P. Aguiar, and C. N. Jones, "Distributed state estimation for discrete-time linear time invariant systems: A survey," Annual Reviews in Control, vol. 48, pp. 36-56, 2019.

[15] S. I. Roumeliotis and G. A. Bekey, "Distributed multirobot localiza- tion," IEEE Transactions on Robotics and Automation, vol. 18, no. 5, pp. 781-795, 2002.

[16] R. Olfati-Saber, "Distributed Kalman filtering for sensor networks," in Proceedings of the 46th IEEE Conference on Decision and Control, 2007, pp. 5492-5498.

[17] A. Molin, H. Esen, and K. H. Johansson, "Scheduling networked state estimators based on value of information," Automatica, 2019, DOI: https://doi.org/10.1016/j.automatica.2019.108578.

[18] S. Andong, W. Yuangang, and Q. Guoqing, "Admissible measurement noise of variance-constrained satisfactory filter," in Proceedings of the 3rd IEEE World Congress on Intelligent Control and Automation, vol. 1, 2000, pp. 106-108.

[19] J. M. Maestre, D. Muñoz de la Peña, and E. F. Camacho, "Wireless sensor network analysis through a coalitional game: Application to a distributed Kalman filter," in Proceedings of the 8th IEEE International Conference on Networking, Sensing and Control, 2011, pp. 228-233.

[20] G.-R. Duan and H.-H. Yu, LMIs in control systems: analysis, design and applications. CRC press, 2013.

[21] P. Gahinet, A. Nemirovskii, A. J. Laub, and M. Chilali, "The LMI control toolbox," in Proceedings of the 33rd IEEE Conference on Decision and Control, vol. 3, 1994, pp. 2038-2041.

[22] X. Yu and K. Tomsovic, "Application of linear matrix inequalities for load frequency control with communication delays," IEEE Transactions on Power Systems, vol. 19, no. 3, pp. 1508-1515, 2004.

[23] Z. Wan and M. V. Kothare, "An efficient off-line formulation of robust model predictive control using linear matrix inequalities," Automatica, vol. 39, no. 5, pp. 837-846, 2003.

[24] S. B. Chabane, C. S. Maniu, T. Alamo, E. F. Camacho, and D. Dumur, "A new approach for guaranteed ellipsoidal state estimation," in Proceedings of the 19th IFAC World Congress, 2014, pp. 6533-6538.

[25] C. Edwards and P. P. Menon, "On distributed pinning observers for a network of dynamical systems," IEEE Transactions on Automatic Control, vol. 61, no. 12, pp. 4081-4087, 2016.

[26] K. Liu, H. Zhu, and J. Lü, "Cooperative stabilization of a class of LTI plants with distributed observers," IEEE Transactions on Circuits and Systems I: Regular Papers, vol. 64, no. 7, pp. 1891-1902, 2017.

[27] G. Casella and R. L. Berger, Statistical inference. Duxbury Pacific Grove, CA, 2002, vol. 2.

[28] L. Vandenberghe, "Positive definite programming," SIAM Review, 1994.

[29] E. L. Lawler and D. E. Wood, "Branch-and-bound methods: A survey," Operations research, vol. 14, no. 4, pp. 699-719, 1966. 